\title{
Classifying Community QA Questions That Contain an Image
}

\author{
Kenta Tamaki \\ Waseda University \\ madao@akane.waseda.jp \\ Sumio Fujita \\ Yahoo Japan Corporation \\ sufujita@yahoo-corp.jp
}

\author{
Riku Togashi \\ Yahoo Japan Corporation \\ rtogashi@yahoo-corp.jp \\ Hideyuki Maeda* \\ CyberAgent, Inc. \\ maeda_hideyuki@cyberagent.co.jp
}

\author{
Sosuke Kato \\ Waseda University \\ sow@suou.waseda.jp \\ Tetsuya Sakai \\ Waseda University \\ tetsuyasakai@acm.org
}

\begin{abstract}
We consider the problem of automatically assigning a category to a given question posted to a Community Question Answering (CQA) site, where the question contains not only text but also an image. For example, CQA users may post a photograph of a dress and ask the community "Is this appropriate for a wedding?" where the appropriate category for this question might be "Manners, Ceremonial occasions." We tackle this problem using Convolutional Neural Networks with a DualNet architecture for combining the image and text representations. Our experiments with real data from Yahoo Chiebukuro and crowdsourced gold-standard categories show that the DualNet approach outperforms a text-only baseline $(p=.0000)$, a sum-and-product baseline $(p=.0000)$, Multimodal Compact Bilinear pooling $(p=.0000)$, and a combination of sum-and-product and MCB $(p=.0000)$, where the $p$-values are based on a randomised Tukey Honestly Significant Difference test with $B=5000$ trials.
\end{abstract}

\section{KEYWORDS}

community question answering; convolutional neural networks; question categorisation

\section{ACM Reference Format:}

Kenta Tamaki, Riku Togashi, Sosuke Kato, Sumio Fujita, Hideyuki Maeda, and Tetsuya Sakai. 2018. Classifying Community QA Questions That Contain an Image. In ICTIR '18: 2018 ACM SIGIR International Conference on the Theory of Information Retrieval, September 14-17, 2018, Tianjin, China. ACM, New York, NY, USA, 4 pages. https://doi.org/10.1145/3234944.3234948

\section{INTRODUCTION}

In Community Question Answering (CQA) sites, posted questions are organised by category so that users can find their desired questions easily. Each CQA site typically has its own hierarchy of mutually exclusive categories, where the top-level categories might be "Entertainment and Hobbies," "News, Politics, International affairs,"

\footnotetext{
*This work was carried out when this author was at Yahoo Japan Corporation.

Permission to make digital or hard copies of all or part of this work for personal or classroom use is granted without fee provided that copies are not made or distributed for profit or commercial advantage and that copies bear this notice and the full citation on the first page. Copyrights for components of this work owned by others than the author(s) must be honored. Abstracting with credit is permitted. To copy otherwise, or republish, to post on servers or to redistribute to lists, requires prior specific permission and/or a fee. Request permissions from permissions@acm.org.

ICTIR '18, September 14-17, 2018, Tianjin, China

(C) 2018 Copyright held by the owner/author(s). Publication rights licensed to ACM. ACM ISBN 978-1-4503-5656-5/18/09 ..\$15.00

https://doi.org/10.1145/3234944.3234948
}

and so on. In Yahoo Chiebukuro ${ }^{1}$, the most widely-used CQA site in Japan (i.e., a Japanese equivalent of Yahoo! Answers), when a questioner posts her question, the site presents her with an automatically selected list of possible categories, from which she can select one and tag her own question with it. Our goal is to automate the problem of assigning a top-level category to a given question, where the question contains not only text but also an image. For example, CQA users may post a photograph of a dress and ask the community "Is this appropriate for a wedding?" where the appropriate category for this question might be "Manners, Ceremonial Occasions." Clearly, this task is more challenging than classifying purely textual questions, and is practically important: in the real CQA data used in our study, approximately $5.4 \%$ of the questions actually contain an image.

We tackle the aforementioned classification problem using Convolutional Neural Networks (CNNs) [5] with a DualNet architecture for combining the image and text representations. Using real data from Yahoo Chiebukuro, we conduct a large-scale question classification evaluation where the categories actually assigned by the questioners are considered to be the gold standard, and a smallerscale experiment where the gold standard is constructed based on the views of crowd workers. The latter experiment shows that the DualNet approach statistically significantly outperforms a text-only and a sum-and-product baselines, Multimodal Compact Bilinear pooling, and a combination of sum-and-product and MCB.

\section{RELATED WORK}

Handling information from multiple modalities has been studied in various fields. Among these studies, Visual Question Answering (VQA) [1, 2], visual grounding [2, 7], and image captioning [6] are closely related to our task in that these tasks all involve handling of both image and text. In VQA, the system is given an image and a question about that image, and is required to output an answer in natural language. In visual grounding, the system is given an image and a natural language description, and is required to return a bounding box within that image that corresponds to the description. In image captioning, the system is given an image, and is required to output a natural language description of that image.

VQA and visual grounding require the understanding of both image and text inputs. However, the input texts in these tasks concern whatever is featured within the input image: for example, an input text for visual grounding may be "(locate) a small white dog (within the picture)" [7]. In contrast, our question classification

\footnotetext{
${ }^{1}$ https://chiebukuro.yahoo.co.jp/
} 


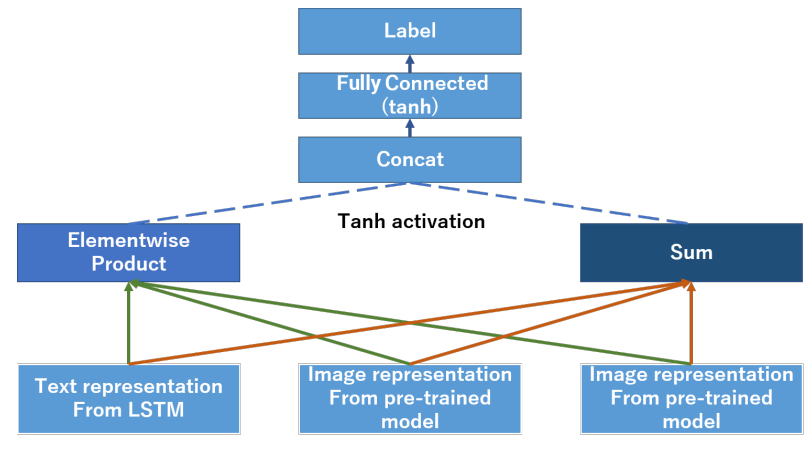

Figure 1: DualNet configuration for real images.

task involves question texts that generally provide a context outside the input image: for example, in the aforementioned example with a photograph of a dress, the accompanying question "Is this appropriate for a wedding?" does not describe any feature within that photograph; rather, it complements the information conveyed in the image, while referring to the dress with the demonstrative pronoun "this." In short, our classification task is different from the aforementioned tasks that involve both text and images.

For VQA and visual grounding, Fukui et al.[2] proposed Multimodal Compact Bilinear (MCB) pooling for joint representation of image and text. The Compact Bilinear pooling model [3] is a technique to compress the high-dimension output of a traditional bilinear pooling model [10]; MCB is a multimodal version of this technique. Fukui et al. argue that MCB complements basic operations such as vector concatenation. In the present study, we apply $\mathrm{MCB}$ to the problem of question classification where each question contains text and an image. Moreover, we explore a combination of MCB with the simple sum and element-wise product approach.

DualNet [8] was proposed in 2016 as a method for VQA, and it outperformed the state of the art in VQA Challenge 2016. Figure 1 depicts the DualNet method in the real images category. In this method, image and text information are combined by fusing the last hidden layer of some pre-trained models. For the real images task, they obtained the text representation from the last hidden layer of LSTM, and the image representation from the hidden layer of multiple pre-trained models. In the present study, we propose to use a model pre-trained with images actually posted to Yahoo Chiebukuro, along with a pre-trained ResNet model [4].

\section{PROPOSED CLASSIFICATION METHODS}

Figure 2 shows our proposed CNN architecture for question classification. The image classification network (top left) is a 5-layer CNN, where the input image size is $128^{*} 128$, with a mini-batch size of 64 . Each convolution layer applies convolution, batch normalization, ReLU activation, and max pooling. Dropout is applied in the fully connected layer.

The text classification network (bottom left) is a character-level CNN [11]. Figure 3 depicts our character-level convolution method. The input text size was set to 121 , which was the average number of characters in our training data. Questions whose text part was longer than 121 characters were excluded from our experiment ${ }^{2}$.

\footnotetext{
${ }^{2}$ It should be noted that 121 characters in Japanese generally conveys much more information than 121 characters in English, as written Japanese relies on ideograms.
}

The vocabulary size (i.e., the number of distinct characters) of the training data was 5,206; accordingly, we set the size of the embedding matrix to $5,208^{*}$ embedding-size (namely, 200), after adding "white space" and "unknown" to the vocabulary. Questions in the validation and test data that were shorter than 121 characters were stuffed with white spaces; characters that never appeared in the training data were treated as "unknown." Our text classification network first prepares an embedding matrix that consists of distributed representations corresponding to each character that can be input. Next, we compute a convolution with a $200^{*}$ height-size*\#channel kernel to the matrix obtained by concatenating the distributed representations of the input characters.

Our final network (Figure 2 middle) utilises pre-trained models of both of the aforementioned networks and a pre-trained ResNet [4] network. Using the hidden layers from the image and text networks, we consider the following four methods for combining the image and text representations (Figure 2 right):

SP Calculate the Sum and element-wise Product; concatenate the results and pass it to the fully connected layer;

MCB Use MCB to generate a joint image and text representation $\left(256^{*} 256 \rightarrow 2,048\right)$; then pass the joint representation to the fully connected layer;

SP+MCB Combination of the above two. As shown in Figure 2, the $\mathrm{MCB}$ representation goes through a fully connected layer and then is concatenated with the Sum and element-wise Product. The result of concatenation is then passed to another fully connected layer.

DualNet Calculate the sum and element-wise Product of text representation, image representation and the hidden layer of a pre-trained ResNet model with tanh activation, and concatenate the results.

\section{CQA AND GROUND TRUTH DATA}

To evaluate our question classification methods, we used real data from Yahoo Chiebukuro. Table 1 shows some statistics of our data: of the approximately $21 \mathrm{M}$ questions, approximately $1.12 \mathrm{M}$ (5.4\%) contain an image; from this set, we extracted 693,519 image-attached questions that cover the ten major top-level categories shown in Table 2. For our experiments, this set was divided into training, test, and validation data sets with the 8:1:1 ratio, and as a result, we obtained 69,355 test questions. By treating the category that is already attached to each test question as the gold standard, we can compute the classification accuracy for each of our methods. Note that, since our problem setting considers ten top-level categories, a random system would only achieve a classification accuracy of $10 \%$ on average.

We argue, however, that the above approach of utilising the actual question category as the gold-standard is not necessarily the best way to evaluate our classification systems. This is because different people may have different views about which category is most appropriate to a given question, and the category assigned by the questioner may not be the same as the one a CQA user, who is looking for an existing question by category, might choose. As the goal of our automatic question classifiers is to provide quick access to CQA users, the views of the CQA users may in fact be more important than that of the questioner. Moreover, the category assigned by the questioner reflects one person's point of view: the 


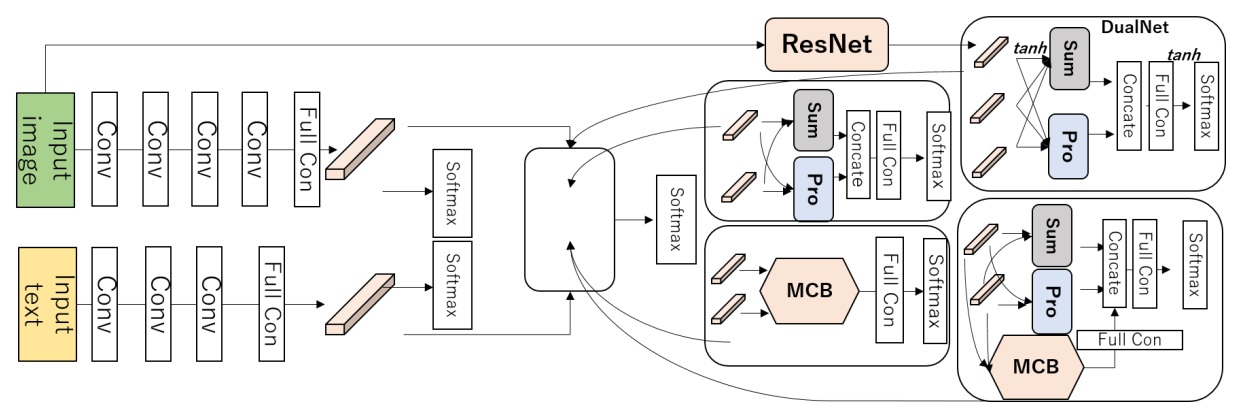

Figure 2: Proposed CNN architecture.

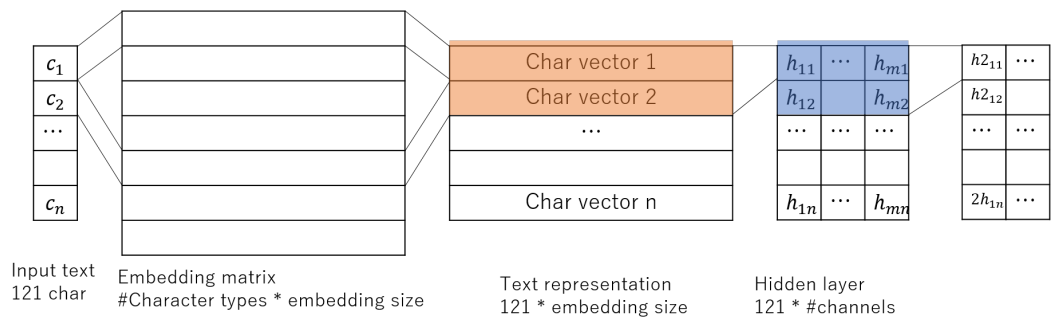

Figure 3: Character level convolution for the text classification network.

Table 1: Number of questions in our CQA data.

\begin{tabular}{|c|r|}
\hline all questions & $20,870,623$ \\
\hline questions with an image & $1,128,164$ \\
\hline $\begin{array}{c}\text { questions with an image } \\
\text { used in the experiment }\end{array}$ & 693,519 \\
\hline training questions & 554,777 \\
\hline test questions & 69,355 \\
\hline $\begin{array}{c}\text { test questions } \\
\text { with crowdsoured category labels }\end{array}$ & 5,190 \\
\hline validation questions & 69,387 \\
\hline
\end{tabular}

Table 2: Ten categories used in our experiment (originally in Japanese).

\begin{tabular}{|l|}
\hline Manners, Ceremonial occasions \\
\hline Entertainment and Hobbies \\
\hline News, Politics, International affairs \\
\hline Internet, PCs, Home appliances \\
\hline Life and romance, Worries of human relations \\
\hline Life, and Living guide \\
\hline Health, Beauty, Fashion \\
\hline Liberal Arts, Learning, Science \\
\hline Sports, Outdoor,Cars \\
\hline Region, Travel, Outing \\
\hline
\end{tabular}

above evaluation methodology cannot take multiple viewpoints into account.

Based on the above argument, we constructed a set of crowdsourced category labels for a subset of the above test questions. Due to budget and time constraints, we randomly selected 519 questions from each category, and thereby constructed a set of 5,190 questions for this second experiment. For each of these questions, five crowd workers were assigned, who independently labeled the questions with a category. They were shown a test question containing an image, and were asked to select the most appropriate category from the list shown in Table 2. They were instructed that the purpose of assigning a category was to enable quick access to the desired question on a CQA site. A total of 205 crowd workers participated in constructing the $5,190 * 5=25,950$ category labels. Rather than deciding on one true category based on a majority vote, we fully utilised the labels from the five assessors as follows. Let $C$ be the set of categories, and let $c_{i} \in C$ be the $i$-th category. Let $\operatorname{votes}\left(q, c_{i}\right)$ be the number of crowd workers $(\leq 5)$ who assigned $c_{i}$ to a given test question $q$. If the system assigns a category $c_{i}$ to $q$, we give $\operatorname{votes}\left(q, c_{i}\right) / 5$ points to the system for $q$. For example, if the system agrees with two assessors for $q$, the partial score for $q$ is $2 / 5=40 \%$.

\section{EXPERIMENTAL RESULTS}

Table 3 summarises our experimental results. It can be observed that, in terms of both classification accuracy and mean partial score, the performance improves as we move down the table.

Table 4 shows the $p$-value for the difference in mean partial score for each system pair (excluding the substantially underperforming image-only baseline) based on a randomised Tukey HSD (Honestly Significant Differences) test with $B=5000$ trials, along with effect sizes (i.e., standardised mean differences) for DualNet computed as recommended in Sakai [9] (Eq. (14)). It can be observed that the DualNet approach outperforms the text-only and sum-and-product baselines, $\mathrm{MCB}$ pooling, and the combination of sum-and-product and $\mathrm{MCB}$, and that the differences are statistically highly significant.

Figure 4 shows confusion matrix heatmaps to visualise the classification accuracy for the text-only (top) and DualNet (bottom) results. The horizontal and vertical axes represent the true (as defined by the questioners) and the predicted categories, and the number of 
Table 3: Experimental results. (a) is based on the correct categories as defined by the questioners. (b) is based on the gold labels obtained from the crowd workers.

\begin{tabular}{|l|r|r|}
\hline & $\begin{array}{r}\text { (a) Accuracy (\%) } \\
(69,355 \text { questions) }\end{array}$ & $\begin{array}{r}\text { (b) Mean partial score } \\
(5,190 \text { questions })\end{array}$ \\
\hline image only & 54.19 & - \\
\hline text only & 73.48 & 0.5114 \\
\hline SP & 77.37 & 0.5165 \\
\hline MCB & 78.00 & 0.5193 \\
\hline SP+MCB & 78.16 & 0.5210 \\
\hline DualNet & 82.86 & 0.5411 \\
\hline
\end{tabular}

Table 4: $p$-values / effect sizes (i.e., standardised mean differences) for the differences in mean partial scores.

\begin{tabular}{|l|r|r|r|r|}
\hline & SP & MCB & SP+MCB & DualNet \\
\hline text only & $p=.7140$ & $p=.2810$ & $p=.1150$ & $p=.0000$ \\
& & & & $E S=.1449$ \\
\hline SP & - & $p=.9652$ & $p=.7924$ & $\begin{array}{r}p=.0000 \\
E S=.1201\end{array}$ \\
\hline MCB & - & - & $p=.9942$ & $\begin{array}{r}p=.0000 \\
E S=.1063\end{array}$ \\
\hline SP+MCB & - & - & - & $p=.0000$ \\
& & & & $E S=.0979$ \\
\hline
\end{tabular}

questions in each cell has been normalised by the number of correct questions for each category. It can be observed that while the text-only network tends to misclassify questions into Category 1 ("Entertainment and Hobbies"), as represented by the light patch in the Category 1 row in the top graph, DualNet does better in this respect. The above tendency of the text-only network is probably due to the fact that we had substantially larger training questions for Category 1 and the network suffered from overfitting towards this category. Whereas, DualNet seems to have alleviated this problem by relying on features from ResNet, a network pre-trained from general and large-scale image corpus.

\section{CONCLUSIONS}

Our experiments with real data from Yahoo Chiebukuro and crowdsourced gold-standard categories show that the DualNet approach statistically significantly outperforms a text-only and a sum-andproduct baselines, Multimodal Compact Bilinear pooling, and a combination of sum-and-product and $\mathrm{MCB}$. While these effects are small (no more than 0.15 in standardised mean difference), the DualNet approach appears to be the most promising for combining image and text representations for question classification.

As we have observed that the categories given by questioners and those given by crowd workers often disagree, we would like to try partially incorporating crowd labels for training our networks in our future work.

\section{REFERENCES}

[1] Stanislaw Antol, Aishwarya Agrawal, Jiasen Lu, Margaret Mitchell, Dhruv Batra, C Lawrence Zitnick, and Devi Parikh. 2015. VQA: Visual question answering. In Proceedings of the IEEE ICCV 2015. 2425-2433.
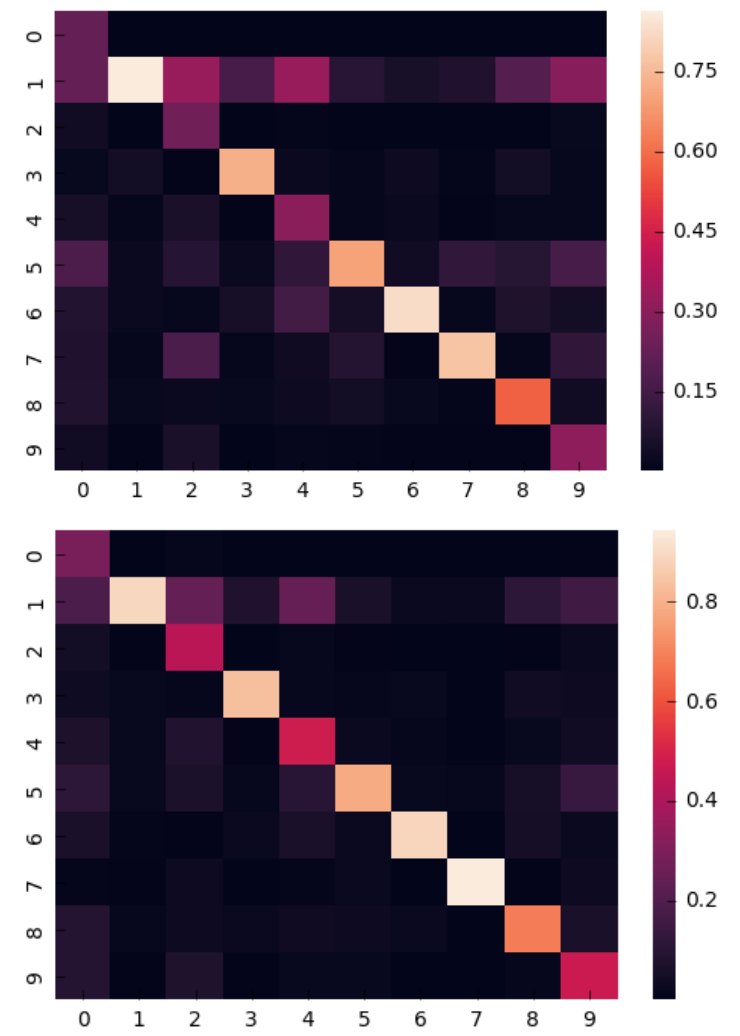

Figure 4: Confusion matrix heatmap. horizontal axis: questioners' categories; vertical axis: text-only categorisation results (top) / DualNet categorisation results (bottom).

[2] Akira Fukui, Dong Huk Park, Daylen Yang, Anna Rohrbach, Trevor Darrell, and Marcus Rohrbach. 2016. Multimodal Compact Bilinear Pooling for Visual Question Answering and Visual Grounding. In Conference on Empirical Methods in Natural Language Processing. ACL, 457-468.

[3] Yang Gao, Oscar Beijbom, Ning Zhang, and Trevor Darrell. 2016. Compact Bilinear Pooling. In Proceedings of IEEE CVPR 2016.

[4] Kaiming He, Xiangyu Zhang, Shaoqing Ren, and Jian Sun. 2016. Deep residual learning for image recognition. In Proceedings of the IEEE conference on computer vision and pattern recognition. $770-778$.

[5] Alex Krizhevsky, Ilya Sutskever, and Geoffrey E Hinton. 2012. Imagenet classification with deep convolutional neural networks. In Advances in neural information processing systems. 1097-1105.

[6] Margaret Mitchell, Xufeng Han, Jesse Dodge, Alyssa Mensch, Amit Goyal, Alex Berg, Kota Yamaguchi, Tamara Berg, Karl Stratos, and Hal Daumé III. 2012. Midge: Generating image descriptions from computer vision detections. In Proceedings of the 13th Conference of the European Chapter of the Association for Computational Linguistics. Association for Computational Linguistics, 747-756.

[7] Anna Rohrbach, Marcus Rohrbach, Ronghang Hu, Trevor Darrell, and Bernt Schiele. 2016. Grounding of Textual Phrases in Images by Reconstruction. In Computer Vision - ECCV 2016, Bastian Leibe, Jiri Matas, Nicu Sebe, and Max Welling (Eds.). Springer International Publishing, Cham, 817-834.

[8] Kuniaki Saito, Andrew Shin, Yoshitaka Ushiku, and Tatsuya Harada. 2017. Dualnet: Domain-invariant network for visual question answering. In Multimedia and Expo (ICME), 2017 IEEE International Conference on. IEEE, 829-834.

[9] Tetsuya Sakai. 2014. Statistical Reform in Information Retrieval? SIGIR Forum 48, 1 (2014), 3-12.

[10] Joshua B. Tenenbaum and William T. Freeman. 2000. Separating Style and Content with Bilinear Models. Neural Comput. 12, 6 (2000), 1247-1283. DOI : http://dx.doi.org/10.1162/089976600300015349

[11] Xiang Zhang, Junbo Zhao, and Yann LeCun. 2015. Character-level convolutional networks for text classification. In Advances in neural information processing systems. 649-657. 